\title{
Late Light Curves of Type Ia SNe
}

\author{
Peter A. Milne ${ }^{1}$ and G. Grant Williams ${ }^{2}$ \\ 1 Steward Observatory, University of Arizona, Tucson, AZ, USA; \\ pmilne@as.arizona.edu \\ 2 MMTO, Tucson, AZ, USA; \\ ggwilli@mmto.org
}

Summary. At late times, the energy deposition in the ejecta of type Ia supernovae is dominated by the slowing of energetic positrons produced in ${ }^{56} \mathrm{Co} \rightarrow{ }^{56} \mathrm{Fe}$ decays. Through comparisons of simulations of energy deposition in SN Ia models with observed light curves from supernovae, we study the positron transport and thus the magnetic fields of SNe Ia. In this paper, we summarize the current status of these investigations, emphasizing the observations made of two recent SNe Ia, 1999by and $2000 \mathrm{cx}$.

\section{Introduction}

The late light curves of Type Ia SNe afford a glimpse into how positrons produced in ${ }^{56} \mathrm{Co} \rightarrow{ }^{56} \mathrm{Fe}$ decays interact with the SN ejecta. As the trajectories of positrons are influenced by magnetic fields, studies of positron transport are equally studies of the magnetic fields in SN ejecta. The essential comparison is between some measure of the energy deposition rate for a SN Ia model and some measure of the luminosity of an observed SN Ia. Implicit in the comparison are the assumptions that the energy deposited in the ejecta is wholly converted into emission and that the observed emission is a reliable tracer of the bolometric luminosity. Whereas the coupling of energy deposition with $100 \%$ re-emission has never been seriously challenged, there is no consensus as to the correct way to estimate the bolometric luminosity.

The earliest works that attempted these comparisons utilized the observations that were available to them. Colgate, Petschek and Kriese [4] compared simulated energy deposition rates with the $\mathrm{B}$ and $\mathrm{V}$ band observations of SNe Ia and concluded that positron escape was in evidence. By contrast, Axelrod in his thesis [1] fit the 700+ day blue spectrum of SN $1972 \mathrm{E}$ with a model that trapped positron energy (he assumed instantaneous, in-situ deposition of $100 \%$ of the positron kinetic energy), but included an infrared catastrophe (IRC, where an increasing fraction of the emission is emitted in an unobservable portion of the IR wavelength range). Axelrod assumed color evolution (where no single band traces the bolometric luminosity) and arrived at one conclusion about positron transport, while Colgate, Petschek and Kriese assumed no color evolution and arrived at a different conclusion. Due to the limitations of our knowledge of the relevant interaction cross-sections, there remains no consensus as to which assumption is correct. 
More recent investigations have attempted to broaden and deepen the comparisons. Ruiz-Lapuente and Spruit [9] emphasized comparisons with UVOIR bolometric light curves (i.e. based on multi-band photometry) and concluded that positron escape was suggested in some, but not all SN Ia light curves. That approach recognized the importance of including a wide wavelength range, but utilized UVOIR bolometric light curves that at late times include only observations made in the principal, BVRI photometric bands (and thus rely upon extrapolations of the UV and IR emission to approximate the bolometric light curve). Cappellaro et al. [3] assumed the $\mathrm{V}$ band is a reliable tracer of the bolometric luminosity ( $a$ la Colgate), and studied a larger number of SN Ia light curves. Their work tested the hypothesis that positron energy deposition drives the light curves at late times, and they concluded that varying degrees of positron escape were suggested by the light curves. Milne, The and Leising ([7], hereafter MTL99) looked at V and B band light curves of $10 \mathrm{SNe}$ Ia and reported that all of the normally- and super-luminous $\mathrm{SNe}$ Ia light curves were consistent with positron escape. The general conclusion from those works was that positrons appear to escape from most $\mathrm{SNe}$ Ia if $\mathrm{V}$ and $\mathrm{B}$ band light curves accurately trace the bolometric luminosity.

\section{BVRI Photometry of 22 SNe Ia}

Milne, The and Leising then extended their first work to include B,V,R and I band photometry of $22 \mathrm{SNe}$ Ia (Milne, The and Leising [8], hereafter MTL01). The efforts were intended to: 1) widen the wavelength range under investigation, and 2) recognize the heterogeneity that exists within the SNe Ia grouping and then to search for trends within sub-classes. The $22 \mathrm{SNe}$ were classified as normally-, sub- or super-luminous based upon their $\Delta \mathrm{M}_{B}(15)$ values and then normalized to have the same luminosity at 65 days (thus studying the shape of the late light curves rather than the absolute magnitudes). They found that the normally-luminous (10) and super-luminous (6) SNe Ia have similar light curve shapes at late times while sub-luminous (6) SNe Ia have different light curve shapes. They further found that the SN color does evolve from 50-175 days. The V band does roughly trace the BVRI bolometric light curves during that epoch for the normally- and super-luminous SNe Ia (hereafter N/SP SNe), but this may be due to a coincidence as the ${ }^{56} \mathrm{Co}$ emission lines give way to ${ }^{56} \mathrm{Fe}$ emission lines. Beyond 175 days, the B,V,R and I band light curves are consistent with no color evolution that follows the energy deposition rate for positron escape.

The sub-luminous SNe Ia studied exhibited light curves with entirely different characteristics than the other two sub-classes. The $\mathrm{V}$ band light curves have more curvature than the energy deposition rates (either assuming positron trapping or positron escape), initially falling faster and later remaining flatter. For the sub-luminous $\mathrm{SNe}$ Ia, the $\mathrm{B}$ band appears to be a 


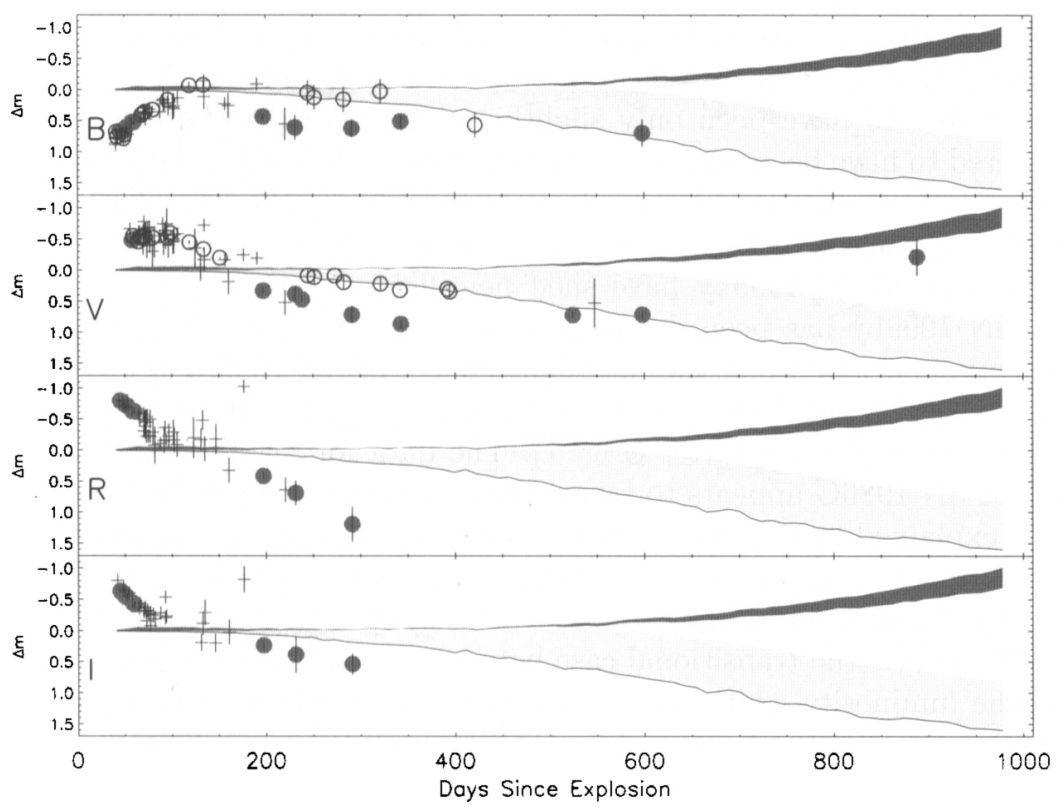

Fig. 1. B,V,R,I band photometry of SN 1999by (filled circles) compared with SN 1986G (open circles) and SN 1991bg (crosses). The data are shown as residuals relative to the energy deposition rates for the model,W7. The light shaded curves assume positron escape, the dark shaded curves assume positron trapping. All SNe were normalized to have the same magnitude at 65 days.

better fit to the energy deposition rates, while the $\mathrm{R}$ and $\mathrm{I}$ band light curves are similar in shape to the other two sub-classes. Derived bolometric corrections appear to be able to explain the color evolution in the light curves of the six SNe Ia, but the number and coverage of the existing spectral data-set are relatively poor and much more work needs to be done before definitive conclusions can be drawn.

\section{Recent Developments}

The previous sections are a review of investigations of the late light curves. The reader is referred to the above publications for details of those investigations. There have been three recent developments that have produced additional interest in this topic. First, the SN 1999by was extensively observed until very late times. Second, SN 2000cx occured with the necessary attributes to be detected two years post-explosion. Third, radiation transport simulations have been performed that include more physics and might provide an alternative explanation for the appearance of positron escape in SN Ia ejecta. 
In MTL01, the SNe 1991bg and 1986G were grouped together as "subluminous" SNe Ia. This classification ignored the distinction that SN 1986G is considered to have been only slightly sub-luminous, while SN 1991bg is considered to have been very sub-luminous (on the low luminosity edge of SN Ia events). That appeared to be adequate considering the irregular sampling of the light curves of those two SNe. However, light curves derived from observations of SN 1999by have shed new light on the sub-luminous subclass. SN 1999by has been determined to be a 1991bg-like based upon the peak emission, and when the late light curves of SN 1999by are compared with SN $1991 \mathrm{bg}$ and $1986 \mathrm{G}$ light curves, it is apparent that 1999 by matches the former and not the latter (Fig. 1). The data for SN 1999by is from [5]. Whereas SN 1986G appears to transition to an epoch where the light curves can be explained by the energy deposition rates that feature positron escape (only B and V band light curves were published), the SNe 1991bg/1999by light curves do not follow the simulations at any epoch. Thus, it can be argued that SN 1986G (the transitional case between normal and fast declining SNe Ia in the luminosity-width relation) transitions at 150 days to light curves similar to the N/SP SNe Ia, while the very sub-luminous SNe Ia follow a different evolution at all epochs. Two SNe should not dictate a distinct subclass, but the shapes of the light curves shown in Fig. 1 do motivate further study of late light curves of sub-luminous SN Ia.

SN 2000cx had peak light curves that challenged the luminosity-width relation by crossing the templates and defying categorization [6]. This fact, combined with the nearness and isolated nature of the SN, made it an ideal target for late-epoch observations. Although the light curves and spectra were unique, they exhibited characteristics spanned by N/SP SNe Ia. Based on that logic, we would expect SN 2000cx's light curves to match the light curves from the $16 \mathrm{~N} / \mathrm{SP}$ SNe studied in MTL01. However, Candia et al. [2]. list a SPF distance to NGC 524 (2000cx's host galaxy) that would make SN $2000 \mathrm{cx}$ a slightly sub-luminous SN Ia, thus the luminosity of SN 2000cx is currently debatable. Shown in Fig. 2 are BVRI light curves of SN 2000cx compared with the $16 \mathrm{~N} / \mathrm{SP}$ SNe Ia. But for an anomaly in the B band from 50-150 days (see also [6]), the shape of the light curves are very similar to the other SNe. The data for SN 2000cx is from Li et al. [6] and Candia et al. [2]. The late data is from Sollerman et al. (these Proceedings), HST R and I band data ( $\mathrm{Li}$ et al., unpublished), and unpublished data we collected at the Steward Observatory $2.3 \mathrm{~m}$ Bok telescope.

The SN 2000cx observations are of additional interest in that Sollerman et al. were able to obtain late $\mathrm{J}, \mathrm{H}$, and $\mathrm{K}$ band photometry for that SN. In a poster shown at this meeting, they report a late flattening of those light curves, potentially due to an IRC. They performed radiation transport for the SN model, W7, and roughly reproduce the IRC while roughly fitting the $\mathrm{V}$ and $\mathrm{R}$ band light curve. Of particular interest is that this fit is accomplished while trapping positrons. Since Fig. 2 shows that the SN 2000cx B, V, R and I 


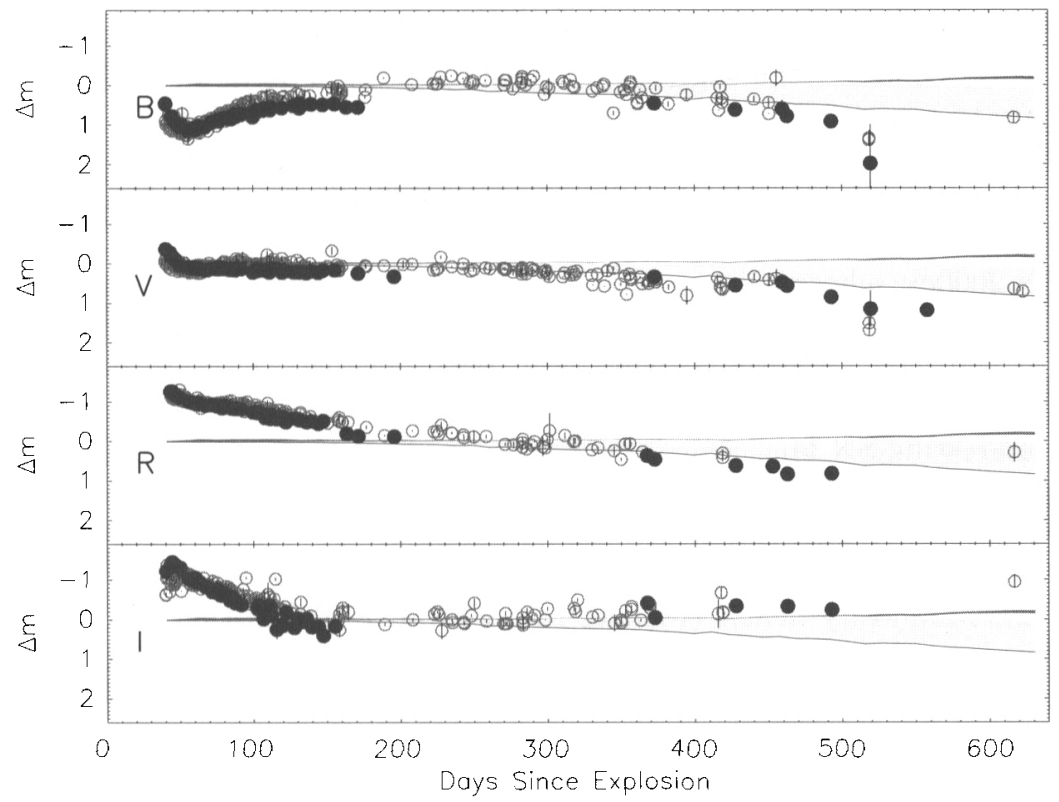

Fig. 2. B,V,R,I band photometry of SN $2000 \mathrm{cx}$ (filled circles) compared with 16 other normally- or super-luminous SNe Ia (open circles). The data are shown as residuals relative to the energy deposition rates for the model W7. The light shaded curves assume positron escape, the dark shaded curves assume positron trapping. All SNe were normalized to have the same magnitude at 65 days.

band light curves agree with other $\mathrm{SNe}$ Ia out to 500+ days, the suggestion is that color evolution in the $\mathrm{B}, \mathrm{V}, \mathrm{R}$ and $\mathrm{I}$ bands might exactly mimic the effects of positron escape. The simulations shown in the Sollerman et al. poster would not be able to explain the SNe that have been detected in the B,V,R and I bands after 600 days (1992A and 1990N for example), nor have they been shown to reproduce SN Ia light curves and spectra in detail. However, the work presents an alternative explanation for the late emission and represents an exciting development in this field.

\section{Summary}

Although challenging to detect, some SNe Ia have been observed until late epochs. From the collection of light curves derived from those observations, a picture is emerging. The late light curves of normally- and super-luminous SNe Ia appear quite similar from 50 - 500 days, appearing as virtually a single set of shapes. By contrast, the sub-luminous SNe Ia have light curves that differ a great deal from the others, and amongst themselves. The two well-observed very sub-luminous SNe 1991bg and 1999by have similar light 
curves whose B and V bands differ appreciably from the N/SP SNe at every epoch. By contrast, the slightly (or transitionally) sub-luminous SN 1986G appears to transition to having light curves that agree with the N/SP SNe Ia. The sub-luminous sub-class is severely under sampled, and it remains an open question whether the late light curves will continuously transition to the N/SP SNe.

SN 2000cx, whose near-peak light curves were unique, appears fairly typical of the N/SP sub-class with respect to the shape of the late light curves out to 400 days. Additional, very late, HST observations were performed, thus it is possible that yet more information can be obtained with regards to this interesting SN Ia.

Interpreting the light curves in terms of positron energy deposition remains an elusive goal. The N/SP SNe Ia are suitably fitted by energy deposition rates that feature positron escape. However, other simulations suggest that the luminosity deficit (relative to positron trapping) that results when positrons escape may instead be due to color evolution that coincidentally mimics the effects of positron escape.

\section{References}

1. T.S. Axelrod: Ph.D. Thesis (Univ. Calif.: Santa Cruz, 1980)

2. P. Candia et al. : Pub. Astron. Soc. Pacific 115, 277 (2003)

3. E. Cappellaro et al. : Astron. Astrophys. 328, 203 (1990)

4. S.A. Colgate, A.G. Petschek, J.T. Kriese: Astrophys. J. Lett. 237, L81 (1980)

5. P. Garnavich et al. : astro-ph 0105490 (2001)

6. W. Li et al. : Pub. Astron. Soc. Pacific 115, 453 (2003)

7. P.A. Milne, L.-S. The, M.D. Leising: Astrophys. J. Suppl. 124, 503 (1999)

8. P.A. Milne, L.-S. The, M.D. Leising: Astrophys. J. 559, 1019 (2001)

9. P. Ruiz-Lapuente, H. Spruit: Astrophys. J. 500, 360 (1998) 